\title{
Was sagt uns Paracelsus heute jenseits der üblichen Klischees?
}

\author{
Johannes G. Schmidt \\ Dr. med., Praxiszentrum Meinradsberg, Präsident des Stiftungsrats "Stiftung Paracelsus Heute»
}

\author{
Auch nach 22 Jahren engagiert sich die Stiftung noch für alternative Zugänge zur \\ Medizin - ganz im Sinne des in Einsiedeln geborenen Arztes Theophrastus Bom- \\ bastus von Hohenheim, bekannt als Paracelsus.
}

Paracelsus war ein Arzt des Mittelalters, das vom Denken der Mystiker wie Thomas von Aquin, Hildegard von Bingen und Meister Eckhart geprägt war. Paracelsus ging es nicht um eine neue Medizin, eher wollte er diese aus der akademischen Sophisterei der Spätscholastik in die Blütezeit des Hochmittelalters zurückführen. Wie auch der Zeitgenosse Luther keine neue Kirche wollte, sondern die Wiederherstellung der Wahrheit des Glaubens und der Religion. Für Luther wie für Paracelsus zählten das eigene Erkennen und die Eingebung Gottes, die über akademische Wahrheiten gingen. Beide erkannten die Gesetze der Selbstheilung und der Gnade, die dem Menschen von der Natur gratis, ohne institu-

Für Luther wie für Paracelsus zählten das eigene Erkennen und die Eingebung Gottes, die über akademische Wahrheiten gingen.

tionelles Zutun gegeben werden. Sie beharrten darauf, dass Ablasshandel und ängstliche Medizin keine richtige Hilfe sind und die Menschen unfrei machen. Geisteskraft und religio lassen sich mit dem erfahrenen klinischen Blick auch heute noch als quasi physiologische Bedingungen erkennen, die die Gesundheit des Menschen bestimmen, auch wenn uns das heute fremd geworden ist - der Schnitt mit dem Mittelalter durch die Moderne ist radikal ausgefallen. Kulturelle Errungenschaften der alten Zeit wie das Zen oder die Tao-Lehre, allerdings, sind durch die Europäische Aufklärung nicht ausgelöscht worden. Die alte chinesische Medizin, die bis heute ungebrochen erhalten ist, spricht vom Yang (Geisteskraft) und von der HerzNieren-Verbindung (Verbindung = religio).

\section{Nachhaltige Heilung und langfristige Gesundheit zählen auch heute}

Das alte kulturelle Wissen, wonach sich Glück in Form von Unglück und Gesundung in Form von Krankheit und Leiden zeigen kann, fordert heraus, die Natural History von Krankheiten und Leiden richtig zu untersuchen. Auch heute brauchen wir eine Wissenschaft der nachhaltigen Heilung und eine physiologische Lehre langfristiger Gesundheit. Man sollte Aufgeklärtsein nicht mit einem Anti-Mittelalter-Reflex verwechseln. Den Aufklärern selbst ging es um die Befreiung aus der selbstverschuldeten Unmündigkeit.

Das moderne medizinische Denken wird auf Dauer die Widerstandskräfte und das Gesundungsvermögen des Patienten nicht ausblenden können, auch wenn sich

\section{Die «Stiftung Paracelsus Heute»}

Die "Stiftung Paracelsus Heute» fördert Bestrebungen, diagnostische, therapeutische und präventive Interventionen der modernen Medizin zu identifizieren und in Frage zu stellen, die lediglich auf theoretisch-akademischen Überlegungen beruhen, ohne dass deren praktischer Nutzen durch kontrollierte Studien belegt ist. Zudem sollen wirksame Heilphänomene und Heilwirkungen identifiziert und kritisch untersucht werden, die auf unkonventionellen Theorie-Systemen beruhen. Dadurch soll die Wissenschaft und Praxis der Medizin erweitert und deren Effizienz gesteigert werden.

Eine besondere Rolle spielt die junge Disziplin der Klinischen Epidemiologie, die als übergreifendes wissenschaftliches Fach- gebiet über den verschiedenen Krankheitstheorien steht. Sie hält die methodischen Grundlagen für eine kontrollierte Beobachtung und empirische Überprüfung von Heilwirkungen bereit.

Die Stiftung ist offen für eine Zusammenarbeit mit geeigneten Universitäten bzw. Universitäts-Instituten. Sie will ihren Zweck verfolgen, indem sie wissenschaftliche Tagungen und eigene Forschung durchführt. Gegebenenfalls regt sie die Gründung wissenschaftlicher Institute und von Institutionen zur Gesundheitsversorgung an.

(Auszug aus den Statuten: http://paracelsus-heute.com/statuten/) 
diese nicht einfach und leicht durch moderne apparative Untersuchungen bestimmen lassen. Mit der Entwicklung der evidenzbasierten Medizin ist sichtbar geworden, dass wir heute oft apparative Untersuchungsergebnisse behandeln, ohne richtige Kenntnis über die Auswirkungen auf den Patienten und seine langfristige Gesundheit zu haben. Pathologien suchen, entfernen oder unterdrücken gleicht schon fast einer abergläubischen Beschäftigung, medizinische Korrektheit herzustellen - gleichsam einer modernen Version des spät-

\section{Das Erkennen und Gewährenlassen der Selbstheilung ist im Grunde das Herzstück einer hochstehenden Medizin.}

mittelalterlichen Ablasshandels. Das Erkennen und Gewährenlassen der Selbstheilung ist im Grunde das Herzstück einer hochstehenden Medizin, wird heute aber als therapeutischer Nihilismus empfunden und als der Fatalismus des dunklen Mittelalters abgelehnt. Die Geisteslehre des Hochmittelalters entspricht freilich dem globalen Erbe, das ausserhalb Europas erhalten geblieben ist und von einigen in China als YangSchule oder Feuerlehre noch heute praktiziert wird: Es sind Kälte-Blockaden, die im Wesentlichen den Fluss im Körper behindern und gleichzeitig die Wahrnehmung über sich selbst anästhesieren und es kalt ums Herz werden lassen. Die Welt ist für den Menschen kalt und dunkel, und so haben die alten Meister und Kulturen weltweit die Pflege des inneren Lichts geübt und der Geisteskraft und Widerstandskraft die Aufmerksamkeit geschenkt. Kälte-Einflüsse führen zu Hitze. Entzündungen oder Fieber, die wir heute bei der Wundheilung oder der Grippe noch gut als Ausdruck der Heilung kennen, lassen sich in der Praxis der Feuerlehre fast generell als Heilungsvorgänge erkennen. Die alte Medizin interessiert sich für den Prozess der Heilung, während die moderne Medizin die natürliche Heilung eher fürchtet, weil sie nicht sieht, dass es oft die Heilung ist, die krank macht - man kann an einem heilenden Grippefieber eben auch sterben, wenn die Widerstandskraft fehlt oder aufgebraucht ist.

\section{Ein erster Schritt: Die «Natural History» von Krankheiten erforschen}

Paracelsus' mittelalterliche Sicht entspricht der altchinesischen Feuerschule, wenn er Krankheiten als Fegefeuer begreift: Der natürliche oder therapeutische Aufbau des inneren Feuers ermöglicht die Regeneration, welche Kälte-Blockaden aufräumt, die die Integrität von Körper und Psyche behindern. Unter modernen Menschen ist eine solche Erweiterung des gesundheit- lichen Verstehens im Gange. Wir können heute eigenwillige Patienten beobachten, die etwa ihre Krebsmetastasen schliesslich aus dem Körper herausfiebern und gesund werden, wenn die Widerstandskraft gestützt wird und hält. Nervenentzündungen bei MS oder Gelenksentzündungen bei Rheuma sind Ausdruck, dass der Körper innere Kälte aufräumt - es sind wiederum Heilungsvorgänge, die krank machen. Nur wenn die Widerstandskraft zu klein ist, erschöpft und erstickt wird, bleibt das Problem chronisch und führt zu einem komplizierten und fatalen Verlauf. Es bleibt die drängende wissenschaftliche Frage: Wie wird man nachhaltig gesund?

Die alte Medizin kennt Mittel, den Geist wach zu machen und die Widerstandskraft zu stärken, was Spontanheilungen begünstigen kann; die moderne Medizin hat Mittel, Krankheiten und ihre Komplikationen einzudämmen, was hilfreich sein kann, wo die Widerstandskraft versagt. Es ist einleuchtend, dass eine gute Balance zwischen dem alten Wissen und den modernen Möglichkeiten uns weiterbringt. Ein erster Schritt ist die bessere Erforschung der Natural History von Krankheiten, sei es durch Studien oder einfach durch sorgfältige Beobachtung in der Praxis. So können wir erkennen, was die Selbstheilung ohne medizinisches Zutun leistet und wie Spontanheilungen zustande kommen. Von Paracelsus lernen wir, dass wir uns nicht allein auf Bücher-Wissen verlassen dürfen, sondern erst durch das Verstehen der Natur das Ganze sehen können. Die Aufklärer machen uns aufmerksam, dass die

\section{Heilungsvorgänge machen krank.}

selbstverschuldete Unmündigkeit das "Mittelalter» in uns ausmacht. Ist dieses dunkle Mittelalter effektiv überwunden? Oder ist uns nur der Zugang zum hellen Mittelalter abhanden gekommen?

\section{Der Wert der klinischen Epidemiologie}

Krankheit steht im Wechselspiel mit dem Gesundungsvermögen. Die Klinische Epidemiologie unterscheidet zwischen wahrem Zusammenhang und Schein-Zusammenhang (Confounding) sowie zwischen wahrem Patientennutzen und Surrogat-Wirkung. Dadurch lässt sich erkennen, dass es Widerstandskraft und Selbstheilung sind, die viele medizinische Erfolge konfundieren und vortäuschen. Pflegt ein Arzt eine unterstützende Haltung dem Patienten-Menschen gegenüber und versteht er auch das kreative Trösten als gute Medizin, oder kennt er weitere Therapien, um das Gesundungsvermögen zu stärken, dann ist er schneller bereit, die ernüchternden wissenschaftlichen Analysen gelten 
zu lassen: Soweit die evidenzbasierte Medizin nämlich nicht glaubt, evident und relevant sei nur, was akademisch korrekt untersucht sei, offenbart sie die Trugschlüsse bei zahlreichen medizinischen Standards. Die internationale Cochrane Collaboration wurde wie die Stiftung 1993 gegründet und zeigt mit ihrer wachsen-

\section{Von Paracelsus lernen wir, dass wir uns nicht allein auf Bücher-Wissen verlassen dürfen.}

den Sammlung systematischer Reviews die Nutzlosigkeit vieler etablierter medizinischer Massnahmen auf (Cholesterinscreening, Krebs-Früherkennung, MSBehandlungen, Grippeimpfung und viele mehr ...). Nicht die Wirksamkeit einer medizinischen Massnahme ist letztlich entscheidend, sondern die tiefergehende Frage, was eine gesunde und nachhaltige Wirkung ist. Die evidenzbasierte Medizin ist eine Denkschule des genauen Hinterfragens und verlangt seit über 20 Jahren eine Orientierung an gesundheitsbezogenen Forschungs-Endpunkten, weil die Wirkung auf eine Krankheit nicht notwendig mit einem Nutzen für den Patienten zusammengeht. Medizinische Wirkungstheorie ist sekundär geworden, das entscheidende Mass ist der effektive, langfristige Vorteil einer Wirkung auf die Fähigkeiten des ganzen Patienten. Mutige Patienten haben begonnen, den Ausgang aus der Unmündigkeit zu wagen. Obwohl heute systematische wissenschaftliche Analysen vorliegen, die den fraglichen Nutzen zahlreicher medizinischer Massnahmen zeigen, ist es für Ärzte noch schwierig, ihre Aufgabe jenseits einer ängstlichen Krankheitsbekämpfung zu erkennen. Mit Hilfe der klinischen Epidemiologie lassen sich die vielen unaufgeklärten medizinischen Denkfehler inzwischen aber sezieren, und das kann Ärztinnen und Ärzte zunehmend befähigen, den Patientennutzen genauer zu hinterfragen und den Ausgang aus einer unüberlegten Absicherungs-Medizin zu finden.

Die Inquisition-lat. Wort für Diagnostik - hat sich erst durch ihre nutzlose Anwendung diskreditiert.

Korrespondenz: Dr. med. Johannes G. Schmidt Praxiszentrum

Meinradsberg

Ilgenweidstrasse 3

CH-8840 Einsiedeln

schmidt[at]

paracelsus-heute.ch

Die wissenschaftlichen Symposien der Stiftung in den 90er Jahren sind legendär. Gleichzeitig hatte die Stiftung hierzulande die ersten Kurse in der kritischen Beurteilung medizinischen Wissens nach den Regeln der evidenzbasierten Medizin ins Leben gerufen (patroniert von der FMH), ebenso wie Lehrgänge in Qigong und Zen. Die Kurse zur Methodik des Hinterfragens der medizi- nischen Literatur werden bis heute in Winterthur weitergeführt.

Mit der Stiftung verbunden ist das Praxiszentrum Meinradsberg, das 1998 am Rande des Klosterplatzes Einsiedeln entstanden ist. Hier wird alte und moderne Medizin kombiniert, wobei der altchinesischen Devise gefolgt wird, wenn möglich nie die Gesundungskräfte zu schädigen durch unnötige Massnahmen wie belastende Medikamente oder Untersuchungen ohne echte therapeutische Konsequenzen. Denn sehr schnell kann des Guten zu viel die Regeneration der Kräfte und damit die Gesundung verhindern. Die richtige «Dosis» ist entscheidend. Die Selbstheilung erkennen und beherzt gewähren lassen, ohne zu übertherapieren und nutzlose Untersuchungen* zu veranlassen, ist und bleibt die zentrale ärztliche Aufgabe. So lassen sich manche der als unheilbar oder tödlich geltenden Krankheiten in einen guten Verlauf überführen; allerdings lässt sich das Leiden oft nicht direkt behandeln und reduzieren, es geht darum, die Kräfte des Patienten geduldig so zu stützen, dass er heil durch das Leiden hindurchkommt, sich selber begreifen lernt und so sein eigener Arzt wird. Unserem Stiftungsrat Luzi Dubs ist in seiner langen Praxis der orthopädischen Chirurgie die Überlegenheit des Spontanverlaufs gegenüber zahlreichen chirurgischen Reparatur-Eingriffen aufgefallen, und er hat massgeblich dazu beigetragen, dass eine Reihe von Operationen heute als nachteilig erkannt worden sind.

Mit medit die andere Sicht auf den Patienten ist durch die Initiative von Jon Boner eine Internet-Plattform am Entstehen, welche durch die Erfassung elektronischer Krankengeschichten einer anderen Art den Blick auf

\section{Mutige Patienten wagen den Ausgang aus der Unmündigkeit.}

die Patientengeschichten lenkt und Daten generiert, welche die Vielfältigkeit der Natural History von Krankheiten beschreiben lassen. Eine intelligente Software erlaubt die Auswertung frei verfasster Einträge, die nicht nur klinisch-apparative Daten, sondern auch klinische Eindrücke und Angaben über die Gesundungskompetenz des Patienten enthalten sollen.
Literatur

- www.paracelsus-heute.ch/cms/praxis/team/PDF/ASA_Vortrag _ Skriptum.pdf

- www.paracelsus-heute.ch/cms/literatur/PDF/SAeZ2O14__NachhaltigeGesundheit.pdf 